\title{
SEISMIC RESPONSE ANALYSIS OF NONPROPORTIONAL DAMPING SYSTEM DUE TO RESPONSE SPECTRUM METHOD
}

\author{
By Yoshikazu YAMADA* and Kenji KAWANO**
}

\begin{abstract}
This paper presents a dynamic response analysis of a nonproportional damping system. Emphasis is placed upon evaluations of seismic response analyses of nonproportional damping systems by means of the response spectrum method. The square-root-sum-ofsquares method causes gross errors which are derived from neglecting the modal coupling effects of the nonproportional damping system with modes of closely spaced frequencies. Accordingly, an alternative modal combination procedure, which provides more accurate responses for the nonproportinal damping system such as situations, is developed. Each modal combination method that is being discussed is evaluated by comparing results with a direct time integration results, i. e. by a Newmak's $\beta$ method.

Keywords: Nonproportional Damping, Response Spectrum, Modal Coupling
\end{abstract}

\section{INTRODUCTION}

In earthquake engineering, a response spectrum method to seismic excitations in generally utilized as the most convienient method. The response spectrum is a function describing the mean of the peak response of an oscillator of the varying frequency and damping to input excitations. Using the response spectrum method to a nonproportional damping system, it is necessary to evaluate the damping ratio and natural frequency of a vibration system, which can be carried out by means of the diagonalization of the nonproportional damping matrix. Several procedures for the diagonalization of the nonproportional damping matrix have been suggested ${ }^{1) \sim 5)}$. For structures with well-separated natural frequencies, it is well known that the square-root-sum-of-squares (SRSS) method for modal responses is practically useful in the evaluation of seismic responses. On the other hand, for structures with modes of closely spaced frequencies, the SRSS method gives gross errors which are derived from the coupling of modal responses which are neglected in this approach. The more accurate response may be performed by using modal combination methods which can be evaluated with weighted coefficients, expressing ir terms of the modal frequencies, modal damping ratios and duration of a seismic motion. An important feature of these formulations is that they mainly depend upon the evaluations of the coupling effects on modal responses. The modal combination method proposed by $\mathrm{E}$. Rosenblueth et al. appears to give good results if the duration of seismic motion is known ${ }^{10)}$. An alternative modal combination method, utilizing the correlation coefficients evaluated by means of a random vibration approach, has been suggested by A. D. Kiureghian ${ }^{9}$. In this formulation, the correlation coefficients give a function of modal frequencies and modal damping rations. A modal combination method, combining a response spectrum method and a complex eigenvalue

* Member of JSCE, Dr. Eng., Professor, Kyoto Unuversity.

** Member of JSCE, Dr. Eng., Associate Professor, Kagoshima University. 
analysis for a nonproportional damping system, has also been suggested by R. Villaverde and M. P. Singh ${ }^{8) .13)}$.

In this study, alternative modal combination methods, which are represented in terms of the generalized damping matrix that are transformed with the classical normal modes, are discussed. By the application of these methods, seismic response analyses of nonproportional damping systems are examined using the response spectrum method. The evaluation of the modal coupling effect on the seismic response analysis is mainly carried out for situations involving modes of closely spaced frequencies.

\section{RESPONSE SPECTRUM METHOD ON NONPROPORTIONAL DAMPING SYS- TEMS}

\section{(1) Direct Evaluations}

In the dynamic response analysis of a structure, the resonse quantities are mainly dependent upon several significant vibration modes. Since the damping matrix of each element for discretized structure generally has a varity of charactristics, the damping matrix of the total system leads to a nonproportional form. When evaluating the damping matrix of such a system, the equation of motion is shown as:

$[M]\{\ddot{x}\}+[C]\{\dot{x}\}+[K]\{x\}=\{F\} \ddot{z}_{g}(t)$

in which $[M],[C]$ and $[K]$ denote the mass, damping and stiffness matrix of a system, respectively. The vector $\{x\}$ and $\{F\}$ denote a displacement vector and a force vector, respectively. $\ddot{z}_{g}(t)$ also denotes an acceleration of input earthquake ground motion. For the vibration system expressed in Eq. (1), the classical normal mode matrix $[\Phi]$ can be determined by means of the eigenvalue analysis of the undamped vibration system. The reduced modal matrix $[\tilde{\Phi}]$ consists of several classical normal modes which have significant effects on the seismic response analysis of the system. Applying the reduced modal matrix, Eq.

(1) can be transformed to :

$$
\{\ddot{q}\}+[\tilde{C}]\{\dot{q}\}+\left[\omega_{j}^{2}\right]\{q\}=\{f\} \ddot{z}_{g}(t)
$$

in which

$$
\left.\begin{array}{l}
{[\tilde{\boldsymbol{\Phi}}]^{T}[M][\tilde{\boldsymbol{\Phi}}]=[1], \quad[\tilde{\boldsymbol{\Phi}}]^{T}[C][\tilde{\boldsymbol{\Phi}}]=[\tilde{C}]} \\
{[\tilde{\boldsymbol{\Phi}}]^{T}[K][\tilde{\boldsymbol{\Phi}}]=\left[\omega_{j}^{2}\right], \quad[\tilde{\Phi}]^{T}\{F\}=\{f\}, \quad\{x\}=[\tilde{\Phi}]\{q\}}
\end{array}\right\}
$$

The generalized damping matrix $[\tilde{C}]$ cannot be generally diagonalized using the reduced modal matrix $[\tilde{\Phi}]$. However, the diagonalization of the nonproportional damping matrix provides a very convenient method for the seismic response analysis. Several procedures of the diagonalization have been suggested. But the most simplified procedure of the diagonalization can be demonstrated by neglecting the off-diagonal terms of a generalized damping matrix $[\tilde{C}]$. Consequently, diagonalized damping matrix is expressed by

$[\tilde{\Phi}]^{T}[C][\tilde{\Phi}] \doteqdot\left[2 \beta_{j} \omega_{j}\right]$

in which $\beta_{j}$ and $\omega_{j}$ denote a damping ratio and a natural frequency of the jth mode. The simplified procedure of the diagonalization is most commonly applicable to the seismic response analysis of the nonproportional damping system. It is, however, necessary to investigate the validity of the application of Eq. (4). Incidentally, the dynamic response analysis for the nonproportional damping system can be performed with the complex eigenvalue analysis. This eigenvalue is determined with twice the degree-of-freedom of the governing equation. In this case, the direct application of the complex eigenvalue analysis to Eq. (1) is not desirable because it is necessary to carry it out with enormous calculations. It is now appropriate to examine the application of the complex eigenvalue analysis for the generalized system with few classical normal modes as expressed in Eq. (2). This equation, which consists only of significant vibration modes, can be easily transformed into a first order differential equation. Applying the complex eigenvalue analysis, Eq. (2) can be expressed by

$$
\{\dot{r}\}+[\lambda]\{r]=\{G\} \ddot{z}_{g}(t)
$$

in which 


$$
\begin{aligned}
& {[\Psi]^{-1}[D][\Psi]=[\lambda], \quad\{G\}=[\Psi]^{-1}\{p\}, \quad\{u\}=[\Psi]\{r\}} \\
& \{u\}=\left\{\begin{array}{l}
\{\dot{q}\} \\
\{q\}
\end{array}\right\}, \quad\{p\}=\left\{\begin{array}{c}
\{f\} \\
\{0\}
\end{array}\right\}, \quad[D]=\left[\begin{array}{cc}
{[\tilde{C}]} & {\left[\omega_{j}^{2}\right]} \\
-[1] & {[0]}
\end{array}\right]
\end{aligned}
$$

The variable $\lambda$ is the complex eigenvalue and $[\Psi]$ is the corresponding complex eigenvector. Now consider the seismic response analysis by means of the response spectrum method. If the nonproportional damping matrix can be diagonalized with available methods, it seems that it would be easy to apply the response spectrum method. However, no exact response of the system can be obtained because the modal coupling effects can not adequately be evaluated with the diagonalization procedures.

On the other hand, using the response spectrum method for the transformed system by means of the complex eigenvalue analysis, the complex response for Eq. (5) can be expressed by

$$
\begin{aligned}
r_{j}= & G_{j} \int_{0}^{t} \exp \left[-\lambda_{j}(t-\tau)\right] \ddot{z}_{g}(t) d \tau=G_{j}\left[\int_{0}^{t} \exp \left[-\omega_{j} \beta_{j}(t-\tau)\right] \cos \left\{\omega_{j}(t-\tau)\right\} \ddot{z}_{g}(\tau) d \tau\right. \\
& \left.+i \int_{0}^{t} \exp \left[-\omega_{j} \beta_{j}(t-\tau)\right] \sin \left\{\omega_{j}(t-\tau)\right\} \ddot{z}_{g}(\tau) d \tau\right] \doteqdot G_{j}(1+i) S_{v}\left(\omega_{j}, \beta_{j}\right) \ldots \ldots \ldots \ldots \ldots \ldots \ldots \ldots \ldots \ldots \ldots \ldots
\end{aligned}
$$

in which $S_{v}\left(\omega_{j}, \beta_{j}\right)$ denotes a velocity respones spectrum value for the damping ratio $\beta_{j}$ and natural frequency $\omega_{j}$, and $i=\sqrt{-1}$. After obtaining the complex response by the specified response spectrum value, the seismic response of the nonproportionl damping system can be evaluated with the tranformed relation of Egs. (6). Then, the generalized response vector $\{q\}$ and $\{\dot{q}\}$ can be provided for the nonproportional damping system. Using these results, the corresponding response vector $\{x\}$ can be determined with the tranformed relation of Eqs. (3). However, since the complex modes have some different effects on the application of the response spectrum method, it is also necessary for the seismic response analysis of the nonpropotional damping system to examine the validity of the application of the complex mode analysis.

\section{(2) Modal Coupling Evaluations}

It is well known that the application of the classical normal mode, which is obtained by the eigenvalue analysis of the undamped vibration system, generally provides available methods for the seismic response analyses. Although the nonproportional damping system cannot be diagonalized with the classical normal modes, its application generally leads to simplified methods on the seismic response analysis. Moreover, as mentioned before, applications of classical normal modes are very effective for the seismic response analysis by means of the response spectrum method. Since a response spectrum method presents the maximum response of each vibrational mode, more exact evaluations of the response of the nonproportional damping system may be provided for considering the modal coupling effects on the responses. This calculation is generally performed with the results on the classical normal mode analysis as expressed in Eq. (3) and Eq. (4). There are several procedures for evaluting the modal coupling effects. But the square-root-sum-squares (SRSS) method is probably the most simplified procedure. Applying the SRSS method, the displacement of some point $n$ of a structure is given by :

$$
x_{n}^{2}=\sum_{j}\left(\tilde{\Phi}_{n j} S_{j}\right)^{2}
$$

in which $\tilde{\Phi}_{n j}$ is the modal value of the $j$-th mode with respect to some point $n$ of a structure, and $S_{j}$ the maximum response value of the $j$-th mode obtained by a response spectrum. On performing the diagonalization of the damping matrix, the SRSS method is approximately applicable to the nonproportional damping system. For the vibration system of well-separated natural frequencies, the SRSS method generally provides good results.

On the other hand, it is observed that the SRSS method often gives significant errors in the case of vibration modes with closely spaced frequencies. For the nonproportional damping system of vibration modes with closely spaced frequencies, it is desirable to provide more accurate and improved methods for the seismic response analysis. Approximate modal combination methods, which take into account for coupling effects of each modal maximum response, are suggested. 
By the application of these methods, the displacement response of the point $n$ of a structure can be generally given by

$$
x_{n}^{2}=\sum_{j} \sum_{k} a_{j k} \tilde{\Phi}_{n j} \tilde{\Phi}_{n k} S_{j} S_{k}
$$

in which the coefficient $a_{j k}$ denotes the coupling effects of modal maximum responses between the $j$-th mode and $k$-th mode. The evaluntion of response obviously depends upon the determination of the coefficient $a_{j k}$. Utilizing the results of the modal coupling evaluation suggested by $\mathrm{E}$. Rosenblueth et al., the coefficient $a_{j k}$ is given by

$$
a_{j k}=\left[1+\left\{\left(\omega_{j}^{\prime}-\omega_{k}\right) /\left(\beta_{j}^{\prime} \omega_{j}+\beta_{k}^{\prime} \omega_{k}\right)\right\}^{2}\right]^{-1}
$$

in which

$$
\omega_{j}^{\prime}=\omega_{j} \sqrt{1-\left(\beta_{j}^{\prime}\right)^{2}}, \quad \beta_{j}^{\prime}=\beta_{j}+2 /\left(\omega_{j} t_{0}\right)
$$

and $t_{0}$ denotes the duration time of input seismic motion. This expression shows that modal coupling effects suddenly increase for situations with modes of closely spaced frequencies. An alternative method of determining the coefficient, $a_{j k}$, by the application of random vibration approach has been suggested by A. D. Kiureghian ${ }^{9)}$. Namely, for the steady state vibration system subjected to a white noise excitation, the coefficient $a_{j k}$ is approximately given by

$$
a_{j k}=\frac{2 \sqrt{\beta_{j} \beta_{k}}\left\{\left(\omega_{j}+\omega_{k}\right)^{2}\left(\beta_{j}+\beta_{k}\right)+\left(\omega_{j}^{2}-\omega_{k}^{2}\right)\left(\beta_{j}-\beta_{k}\right)\right\}}{4\left(\omega_{j}-\omega_{k}\right)^{2}+\left(\omega_{j}+\omega_{k}\right)^{2}\left(\beta_{j}+\beta_{k}\right)^{2}}
$$

This expression is independent of the duration time of input seismic motion. Since the coefficients depend only upon the modal damping ratios and modal frequencies for the nonproportional damping system, it is convienient to evaluate the modal coupling effects on the seisimc responses. It is also indicated that these modal combination methods, which are associated with a weighted evaluation of modal responses, provide good results for the situations of vibration modes with the closely spaced frequencies.

For the seismic response analysis of the nonproportional damping systems by means of the classical normal modes, a more convienient and effective method is suggested for the evaluation of the coupling effects of modal maximum responses. Now, considering the rms response evaluation by Eq. (2), the modal coupling effects are represented with the generalized damping matrix $[\tilde{C}]$. The modal coupling effects between the $j$-th mode and $k$-th mode are generally proportional to $\tilde{C}_{j k}$ from the results of Eq. (2). It is considered that this represents weighted factors corresponding to the modal coupling effects. The effects may then be expressed with the weighted factors derived by the generalized damping matrix. The modal coupling effects of modal maximum responses between the $j$-th mode and the $k$-th mode may be expressed by

$$
a_{j k}=\left|\tilde{C}_{j k}\right| / \sqrt{\tilde{C}_{j j} \tilde{C}_{k k}}
$$

in which $\tilde{C}_{j k}$ is the element of the generalized damping matrix $[\tilde{C}]$, denoting a modal coupling effect between $j$-th mode and $k$-th mode. Using results of numerical analyses which will be shown later, this relation is adequately applicable to evaluate the modal coupling effects. Since only a few predominant vibration modes contribute to the seismic response quantites, Eq. (12) seems to be available for the evaluations of the modal coupling effects.

Moreover, the evaluations of the modal coupling effects can be developed using the results on the complex eigenvalue analysis as expressed in Eq. (5). Now, considering the case that the input excitation $\ddot{z}_{g}(t)$ may be expressed in terms of a white noise excitation with zero mean, the covariance matrix of a vector $\{r\}$ can be determined by the application of the so-called "moment technique". This covariance matrix of a vector $\{u\}$ can be easily transformed into the covariance matrix of a vector as expressed in Eq. (6). Then the covariance matrix $\left[R_{q q}\right]$ of the generalized response $\{q\}$ can be obtained using these results. Since the complex modes have phase differences for the application of the response spectrum method, the most desirable response may be evaluated with a modal combination method which represents the modal coupling effects. Then, applying the results, the coefficient $a_{j k}$ is given by 


$$
a_{j k}=\left|R_{q_{j} q_{k}}\right| / \sqrt{R_{q_{j} q_{k}} R_{q_{j} q_{k}}} \text {. }
$$

in which $R_{q_{j} q_{k}}$ denotes the convariance response between the $j$-th mode and the $k$-th mode to a white noise excitation. The modal coupling generally has the significant effect on the seismic responses of the nonproportional damping system with modes of closely spaced frequencies. Therefore, the suitable evaluation of modal coupling effects provides good results for seismic responses of the nonproportional damping system.

\section{NUMERICAL RESULTS}

\section{(1) Modal Coupling Effects}

On performing the dynamic response analysis for the nonproportional damping system with the classical normal modes, the evaluations of natural frequencies and modal damping ratios have generally shown significant effects. It is important to precisely evaluate these values. Fig. 1 shows the vibration modal of the nonproportional damping system used in this example. For this modal, consider that the mass $\boldsymbol{M}_{\boldsymbol{j}}(\boldsymbol{j}=$ $1, \cdots, 4)$ and spring constant $K_{j}(j=1, \cdots, 4)$ are constants, and the mass $M_{p}$ and spring constant $K_{p}$ are variables. The ratio of $M_{\rho}$, and $M_{j}, m=M_{p} / M_{j}$, and the ratio of $K_{p}$ and $K_{j}, k=K_{p} / K_{j}$, are assumed to be the parameters. For the damping $C_{j}(j=1, \cdots, 4)$ which is proportional to the $K_{j}$, the modal damping ratio of the first vibration mode is assumed to be $2 \%$. Moreover, the damping ratio $\beta_{p}$ between $10 \%$ and $20 \%$ is assumed for the damping of $C_{p}$. At first examine the evaluation of natural frequencies for the nonproportional damping system of $\beta_{p}=20 \%$. Fig. 2 shows the relations between natural frequencies and the mass ratio $m$ for the stiffness ratio of $k=20$ and $k=40$, respectively. The abscissa denotes the mass ratio $m$ and the ordinate the corresponding natural frequencies. The solid line represents the results from the eigenvalue analysis of the undamped system and the dashed line represents the results from the complex eigenvalue analysis as expressed in Eq. (5). For the nonproportional damping system with modes of closely spaced frequencies, it is shown that these methods give good results for the evaluation of natural frequency. Therefore, the natural frequency of a nonproportional damping system may well be calculated by means of the eigenvalue analysis of the undamped vibration system.

On the other hand, it seems that the determination of modal damping rations has significant effects on the dynamic response analysis. For the nonproportional damping system of modes with closely spaced frequencies, good evaluations are not always provided with the diagonalization procedures because the modal coupling effects have significant contributions to the evaluations of modal responses. Therefore, it is important for the nonproportional damping system to clarify the modal coupling effects on the dynamic

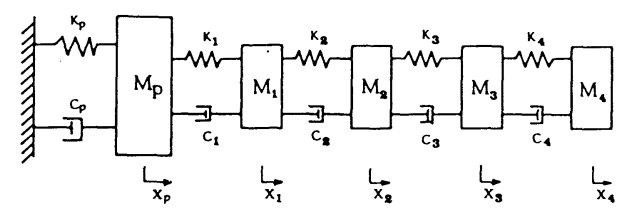

Fig. 1 Vibration Model of Nonproportional Damping Model.
Fig. 2 Natural Frequencies vs Mass Ratio on the Nonproportional damping System.

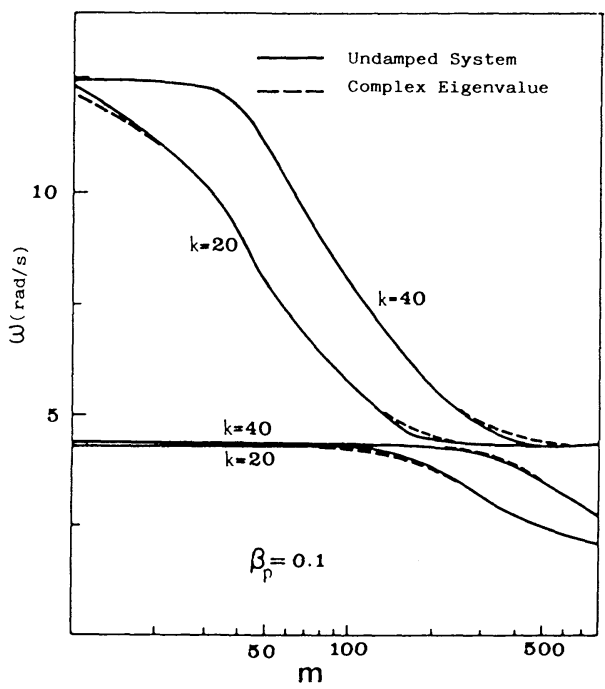


response analysis. Now examine the modal coupling effects by means of the simplified diagonalization procedure as expressed in Eq. (4) and the modal coefficient in Eq. (12). The condition of the diagonalization, which allows for neglecting the off-diagonal terms of the generalized damping matrix $[\tilde{C}]$, has been suggested by G. B. Warburton ${ }^{11)}$. The relationship between the diagonal term and off-diagonal term of the generalized damping matrix $[\tilde{C}]$, are expressed by

$$
\beta_{j}<D_{r}
$$

in which

$$
D_{r}=\varepsilon_{0}\left|\left(\frac{\tilde{C}_{j j}}{\tilde{C}_{j k}}\right)\left\{\left(\frac{\omega_{k}}{\omega_{j}}\right)^{2}-1\right\}\right|_{\text {min }}
$$

The varible $\varepsilon_{0}$ is an arbitrary positive constant and was given by the valus of 0.05 for this study 0.05 is chosen. Fig. 3 shows the relation between the damping ratio of the first mode $\beta_{1}$ and the value $D_{r}$ for the nonproportional damping system of $\beta_{p}=10 \%$ and $k=40$. The abscissa denotes the mass ratio of $\boldsymbol{m}=$ $\boldsymbol{M}_{p} / \boldsymbol{M}_{j}$, and the ordinate the damping ratio (the soild line) and $D_{r}$ (broken line), respectively. The broken-dotted line denotes the modal coefficient of Eq. (12), which represents the modal coupling effects for the first and the second modes. It appears that Eq. (14) not always provide good evaluations for the condition of the diagonalization of the nonproportional damping matrix. On the other hand, using the modal coefficients of Eq. (12) for significant modes, the modal coupling effects on responses may be calculated in the situations with modes of closely spaced frequencies. In the case of the seismic response analysis of nonproportional damping system, it is anticipated that the modal coefficient expressed in Eq. (12) provides a good indicator for the evaluation of modal coupling effects.

\section{(2) Seismic Response Analysis of Nonproportionl Damping System}

The application of classical normal modes is very convenient and effective for the seismic response analysis of the nonproportionl damping system by response spectrum methods as mentioned before. From the practical point of view, when using the seismic response analysis, it is important to investigate the accuracy of the evaluation by means of the modal coupling effects. In paticular it appears that these methods have significant effects on responses of the nonproportionl damping system in situations involving modes with closely spaced frequencies. The seismic response analyses for the nonproportional damping system are then carried out using the response spectra which have been determined for the seismic ground motions of EL CENTRO 1940 NS, and TAFT 1952 N21E components, respectively. The evaluation of the modal coupling effects on the response in such situations is now examined with comparisons of the results from the response spectra and the results from Newmark's $\beta$ method for these excitations.

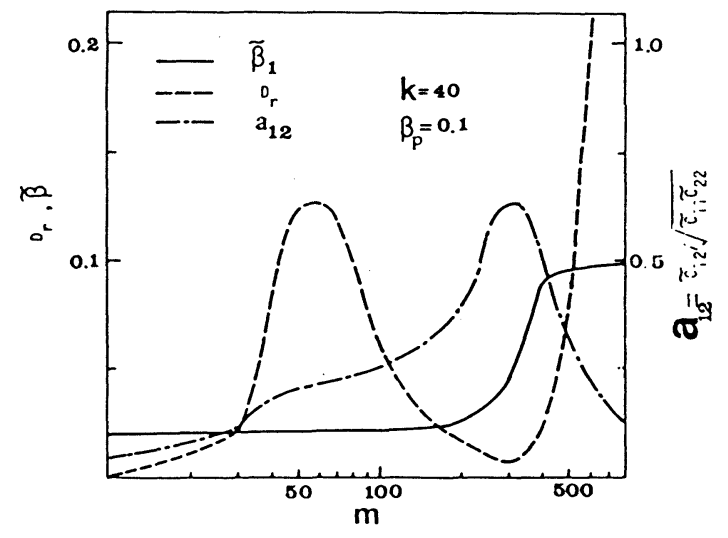

Fig. 3 Evaluations of Diagonalization and Modal Coupling Effects.

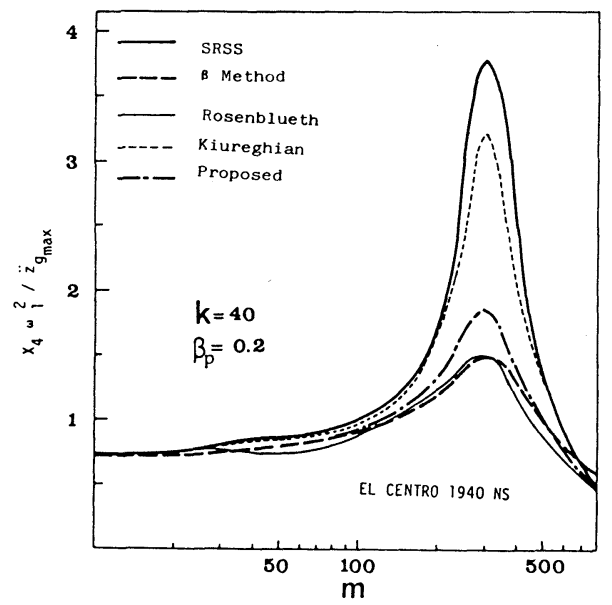

Fig. 5 Maximum Response vs Mass Ratio on the Nonproportional Damping System $(k=20)$. 

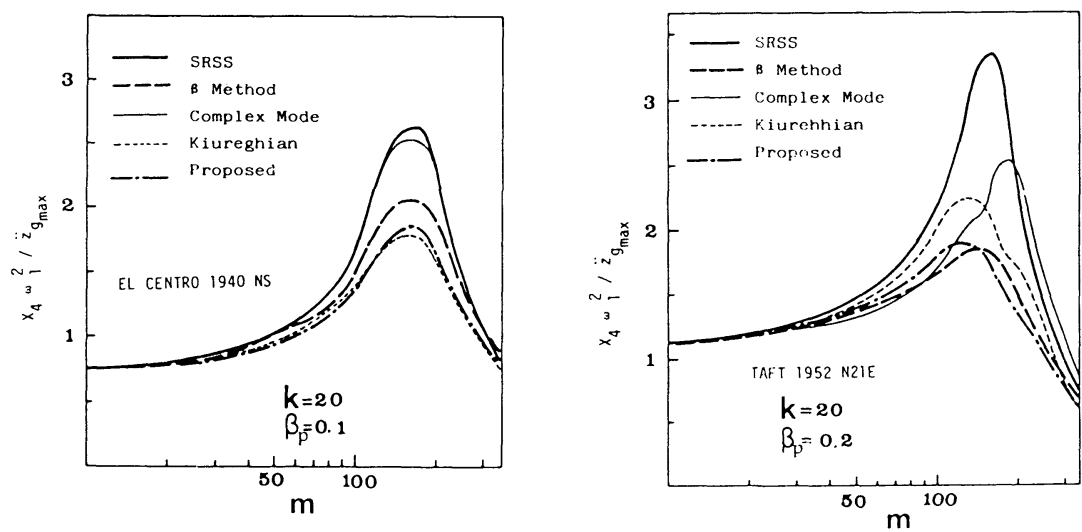

Fig. 4 Maximum Response vs Mass Ratio on the Nonproportional Damping System $(k=20)$.

Fig. 4 shows the relation between the maximum displacement of point $M_{4}$ and the mass ratio for the nonproportional damping system of $\beta_{p}=10 \%$ and $20 \%$. The abscissa denotes the mass ratio $\boldsymbol{m}=\boldsymbol{M}_{p} / \boldsymbol{M}_{j}$ and the ordinate denotes the nondimensional form of the corresponding maximum displacement which is expressed in terms of $x_{4} \omega_{1}^{2} / \ddot{z}_{g \max }$. The variable $\ddot{z}_{g \max }$ denotes the maximum acceleration of the input excitation and $\beta_{1}$ the natural frequency of the first mode. The solid line denotes the results from the SRSS method as expressed in Eq. (8). Each modal damping ratio, required for the determination of the corresponding response spectrum values, is evaluated with the most simplified diagonalization procedure as expressed in Eq. (4). The thin line denotes the results from the SRSS method which is calculated with the damping ratios and natural frequencies determined by the complex eigenvalue analysis. The broken line denotes the results from Newmark's $\beta$ method. The evaluation on the modal coupling effects is done by Eq. (11) for the dotted line and Eq. (12) for the broken-dotted line, respectively. In the situation of the closely spaced natural frequencies between the first mode and the second mode, the modal coupling effect leads to the increase of responses. Moreover, the responses have somewhat different properties for the two input excitations. According to the evaluation of the modal coupling effect, the responses in the situation with modes of closely spaced frequencies present clearly different values. The SRSS method using the most simplified diagonalization procedure generally presents the largest response. Utilizing an alternative diagonalization procedure, the response due to the SRSS method has larger value in comparison with the result from Newmark's $\beta$ method. On the other hand, the responses obtained by taking into account the modal coupling effects, give good agreement for the results from Newmark's $\beta$ method. In the situations with modes of closely spaced frequencies, these modal colbination methods provide somewhat different properties on the evaluations of responses. It is, therefore, necessary for the seismic response analysis to examine not only the SRSS method by means of diagonalization procedures, but also by the modal combination methods.

Fig. 5 also shows the relation between the maximum displacement of point $M_{4}$ and the mass ratio for the nonproportional damping system $\beta_{p}=20 \%$ and $k=40$. The definition of the axis of coordinates is the same as shown in Fig. 4. The solid line presents the results from the SRSS method as expressed in Eq. ( 8 ). Each modal damping ratio, which is used for the evaluation of the response spectrum value, is calculated with the most simplified diagonalization procedure. The thin line gives the results from the modal combination method which is evaluated with the modal coefficients as expressed in Eq. (10). The broken line denotes the results from Newmark's $\beta$ method. The responses, which are calculated by the modal combination methods utilizing the modal coefficients as expressed in Eq. (11) and Eq. (12), denote the dotted line and broken dotted line, respectively. The increase of the response in the situations involving the modal coupling was due to the first mode and the second mode. The SRSS method generally presents 
larger responses than the results from Newmark's $\beta$ method. On the other hand, it is shown that the responses due to the modal combination methods generally provide good evaluations in the situations with modes of closely spaced frequencies. The response, which is calculated with the modal coefficient of Eq. $(10)$, is also partially different from the results due to Newmark's $\beta$ method. Consequently, it is noted that according to the properties of input excitations and the damping ratio $\beta_{p}$, the responses due to the modal combination methods provide somewhat different evaluations in the situations with modes of closely spaced frequencies.

\section{(3) Seismic Response Analysis of A Soil-Structure System}

It is well known that the dynamic soil-structure interaction has significant effects on the response evaluations. The dynamic soil-structure interaction system generally has the nonproportional damping characteristics. Therefore it is necessary for the seismic response analysis of the dynamic soil-structure interaction system to investigte the modal coupling effects on the response evalutions. Fig. 6 shows the soil-offshore structure modal, having the embedded foundation into the seabed soil. Evaluating the dynamic properties of the soil-foundation with the impedance functions, the governing equation can be obtained by the simplified substructure method developed by the authors ${ }^{7}$. The governing equation of motion for this system is represented with the same form as expressed in Eq. (1). Since the damping matrix becomes the nonproportional form, the dynamic response propertis are examined with the procedures as mentioned before.

Fig. 7 shows the relation between the natural frequencies and shear wave velocity of seabed soil. The soil consists of a two-layer stratum which the shear rigidity ratio of the surface stratum and bottom stratum is 0.1 . The solid line represents the results for the radius of foundation $R=45 \mathrm{~m}$ and the embedded depth $h=5 \mathrm{~m}$, and dotted line also represents the results for the radius of foundation $R=50 \mathrm{~m}$ and the embedded depth $h=10 \mathrm{~m}$. It is shown that the closely spaced natural frequencies for the first few vibration modes are caused by conditions of the shear wave velocity between $200 \mathrm{~m} / \mathrm{s}$ and $350 \mathrm{~m} / \mathrm{s}$. For the dynamic response analysis due to the application of the response spectrum method, it seems that it is important to clarify the modal coupling effects on the response evaluations in such situations.

Fig. 8 shows the relations between the maximum displacement of point 19 at the superstructure and the shear wave velocity of the seabed stratum. The solid line denotes the results from the SRSS method as expressed in Eq. ( 8 ). The thin line, dotted line and broken-dotted line also denote the results from the modal combination methods which are evaluated with the coefficients as expressed in Eq. (10), Eq. (11) and Eq. (12), respectively. The broken line denote the results from Newmark's $\beta$ method. The significant modal coupling effects on the response are presented in the shear wave velocity condition which yields the coupling between the predominant frequencies. Applying the modal combination methods with the

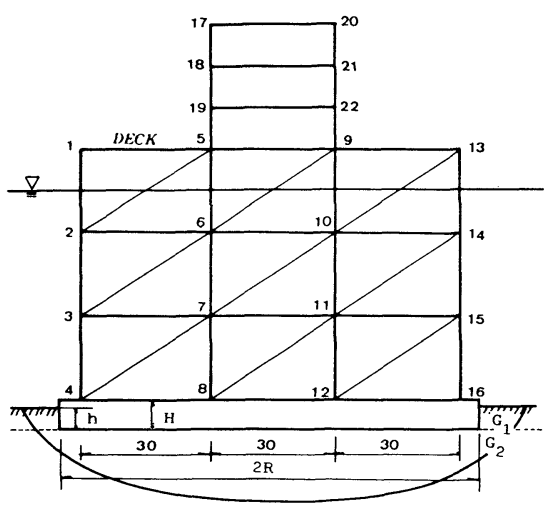

Fig. 6 Soil-Offshore Structure Model.

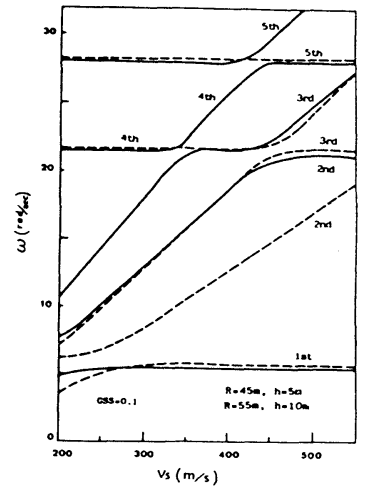

Fig. 7 Natural Frequencies vs Shear Wave Velocity on Soil-Offshore Structure Model. 

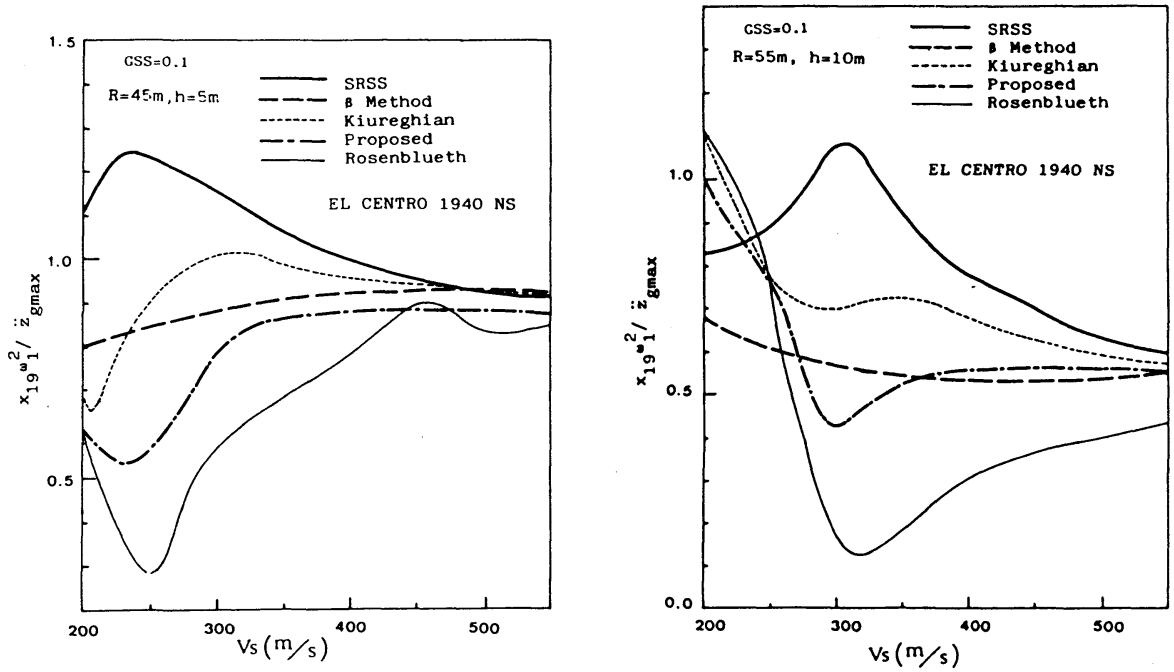

Fig. 8 Maximum Response vs Shear Wave Velocity on A Soil-Offshore Structure Model.

coefficients as expressed in Eq. (11) and Eq. (12), the responses provide better agreement than the results from the SRSS method. The evaluations of responses have somewhat different characteristics for the variations of the dynamic soil-structure interaction. Consequently, it is noted that although the modal combination methods generally provide good evaluations of the modal coupling effects on the responses, more examinations are necessary for the situations with modes of the closely spaced frequencies.

\section{CONCLUSIONS}

The principal results and conclusions of this study can be summarized as follows:

(1) The evaluations of the modal coupling effects have important roles on the seismic response analysis of the nonproportionl damping system by means of the response spectrum method. The modal coupling evaluations on the seismic response are then examined.

(2) The alternative modal combination method is developed using the generalized damping matrix, which is expressed with the reduced damping matrix derived with the modal matrix of the undamped vibration system. It is shown that the weighted coefficient determined with the generalized damping matrix also provides a good indicator for the evaluations of modal coupling effects on the seismic response analysis.

(3) In the situations with modes of closely spaced frequencies, while the response due to the SRSS method using the diagonalization procedures generally seems to produce gross errors, the application of the modal combination methods representing the modal coupling effects generally provide the good results.

(4) The alternative modal combination method provides good results in the seismic response evaluation by means of the response spectrum method. Moreover, for the nonproportional damping system in the situations with modes of closely spaced frequencies, it should be nothed that the modal combination methods generally allow somewhat different evaluations of responses for input excitations and damping properties.

Moreover, since the dynamic stress resultant response characteristics generally seems to be different from the results of the displacement, it would be also necessary to investigate the modal coupling effects on the responses.

\section{REFERENCES}

1) Roesset, J. M., Whitman, R. V. and Dobry, R. : Modal Analysis for Structure with Foundation Interaction, J. Structural 
Division, ASCE, Vol. 99, pp. 399 415, 1973.

2) Tsai, N. C. : Modal Damping for Soil-Structure Interaction, J. Engng. Mech. Division, ASCE, Vol. 100, pp. 323 341, 1974.

3) Yamada, Y. and Takemiya, H. : Random Response Analysis of Multi-Degree-of-Freedom Systems with Non-Proportional Damping and Its Application to the Tower-and-Pier System of Long-Span Suspension Bridge, Proc. of Japan Earthq. Engng. Symposium, Tokyo, pp. 373 380, 1973.

4) Yamada, Y. and Kawano, K. : Dynamic Response Analysis of Systems with Nonproportional Damping, Proc. of the Fourth Japan Earthq. Engng. Symposium, Tokyo, pp. 823 830, 1975.

5) Clough, R. W. and Mojtahedi, S. : Earthquake Response Analysis Considering Non-proportional Damping, Earthq. Engng. and Struc. Dyn. Vol. 4, pp. 489 496, 1976.

6) Clough, R. W. : Analysis of Strutural Vibrations and Dynamic Response, Recent Advances on Matrix Methods in Structural Analysis and Design, Proc. of U. S. -Japan Seminar, The University of Alabama Press, Huntsville, Alabama, 1971.

7) Yamada, Y., Takemiya, H. and Kawano, K. : Random Response Analysis of a Non-Linear Soil-Suspension Bridge, Earthq. Engng. and Struc. Dyn., Vol. 7, pp. 31 47, 1979.

8) Villaverde, R. : Earthquake Response of Systems with Nonproportional Damping by the Conventional Response Spectrum Method, Proc. of the Seventh World Conference on Earthquake Engineering, Istanbul, Turkey, Vol. 5, pp. 467 474, 1980.

9) Kiureghian, A. Der. : A Response Spectrum Method for Random Vibration Analysis of MDF system, Earthq. Engng. and Struc. Dyn., Vol. 9 pp. 419 435, 1981.

10) Rosenblueth, E. and Elorduy, J. : Responses of Linear Systems to Certain Transient Disturbances, Proc. Fourth World Conf. of Earthq. Engng., Vol. 1, Santiago, Chile, pp. 185 195, 1965.

11) Warburton, G. B. and Tsai, S. R. : Errors in Response Calculations for Non-Classically Damped Structures, Earthq. Engng. Struc. Dyn. Vol. 5, pp. 365 376, 1977.

12) Yamada, Y. and Kawano, K. : Effects of Damping Matrices on Dynamic Response Analyses, Proc. of JSCE, No. 334, pp. 43 51, June 1983.

13) Singh, M. P. : Seismic Response by SRSS for Nonpropotional Damping, J. Engng. Mech. Division, ASCE, Vol. 106, EM6, pp. 1405 1419, 1980.

(Received June 27 1986) 